\title{
Age-dependent alterations in the immunoreactivity of macrophage inflammatory protein-3 $\alpha$ and its receptor CCR6 in the gerbil hippocampus
}

\author{
JI HYEON AHN ${ }^{1,2^{*}}$, JOON HA PARK $^{3 *}$, TAE-KYUNG LEE ${ }^{1}$, GO EUN YANG ${ }^{4}$, \\ MYOUNG CHEOL SHIN ${ }^{5}$, JUN HWI CHO ${ }^{5}$, MOO-HO WON ${ }^{2}$ and CHOONG-HYUN LEE ${ }^{6}$
}

${ }^{1}$ Department of Biomedical Science, Research Institute of Bioscience and Biotechnology, Hallym University, Chuncheon, Gangwon 24252; ${ }^{2}$ Department of Neurobiology, School of Medicine, Kangwon National University, Chuncheon, Gangwon 24341; ${ }^{3}$ Department of Anatomy, College of Korean Medicine, Dongguk University, Gyeongju, Gyeongsangbuk 38066; ${ }^{4}$ Department of Radiology, Kangwon National University Hospital, Chuncheon, Gangwon 24289;

${ }^{5}$ Department of Emergency Medicine and Institute of Medical Sciences, Kangwon National University Hospital, School of Medicine, Kangwon National University, Chuncheon, Gangwon 24289; ${ }^{6}$ Department of Pharmacy, College of Pharmacy, Dankook University, Cheonan, Chungcheongnam 31116, Republic of Korea

Received January 5, 2020; Accepted May 21, 2020

DOI: $10.3892 / \mathrm{mmr} .2020 .11216$

\begin{abstract}
Neuroinflammation is a primary characteristic of the aging brain. During normal aging, macrophage inflammatory protein-3 $\alpha$ (MIP-3 $\alpha$ ) and its receptor $\mathrm{C}-\mathrm{C}$ chemokine receptor type 6 (CCR6) serve pivotal roles in the neuroinflammatory process in the brain. The aim of the present study was to investigate age-dependent alterations in the immunoreactivity of MIP-3 $\alpha$ and CCR6 in the gerbil hippocampus at postnatal month (PM) 1, 6, 12 and 24 via immunohistochemistry. In the PM 1 group, both MIP-3 $\alpha$ and CCR6 immunoreactivity were observed primarily in the stratum pyramidale in the hippocampus proper and in the granule cell layer in the dentate gyrus. In the PM 6 and PM 12 groups, MIP-3 $\alpha$ in the stratum pyramidale and granule cell layer was decreased compared with the PM 1 group, and CCR6 immunoreactivity in both layers was faint. In the PM 24 group, MIP-3 $\alpha$ expression in the stratum pyramidale and granule cell layer was higher than that in the PM 1 group, and CCR6 immunoreactivity in both layers was increased compared with the PM 12 group; however, it
\end{abstract}

Correspondence to: Professor Moo-Ho Won, Department of Neurobiology, School of Medicine, Kangwon National University, 1 Gangwondaehak-gil, Chuncheon, Gangwon 24341, Republic of Korea E-mail: mhwon@kangwon.ac.kr

Professor Choong-Hyun Lee, Department of Pharmacy, College of Pharmacy, Dankook University, 119 Dandae-ro, Cheonan, Chungcheongnam 31116, Republic of Korea

E-mail: anaphy@dankook.ac.kr

*Contributed equally

Key words: neuroinflammation, macrophage, aging, hippocampus, pyramidal cells, granule cells was decreased compared with the PM 1 group. In conclusion, MIP-3 $\alpha$ and CCR6 immunoreactivity were altered in the hippocampus during normal aging. The results of the current study suggested that age-dependent alterations of MIP-3 $\alpha$ and CCR6 may be associated with age-related neuroinflammation in the hippocampus.

\section{Introduction}

The hippocampus serves major roles in learning and memory and has been indicated to be the most susceptible brain region during normal aging $(1,2)$. During the aging process, numerous neuropathological and neurobiological alterations occur in the hippocampus, such as induction of the neuroinflammatory process, alterations in synaptic plasticity, a reduction in the ability of protein repair and a decrease in neuronal activity (3-6).

Chemokines serve diverse roles in tissue homeostasis and immune reactions (7). It has been reported that various chemokines and chemokine receptors were widely expressed in neurons and glial cells of the central nervous system (CNS), and they were indicated to function as critical regulators in intercellular communication in the CNS under normal or pathological conditions including stroke, Alzheimer's disease and multiple sclerosis (8-11). Among chemokines, macrophage inflammatory protein-3 $\alpha$ (MIP-3 $\alpha)$, which is also known as C-C motif chemokine 20, has been indicated to interact specifically with its unique receptor, $\mathrm{C}-\mathrm{C}$ chemokine receptor (CCR) type 6 (CCR6) (12). A number of studies have reported that CCRs, including CCR6, were expressed in hippocampal neurons, and they were considered to serve as protective factors that are associated with neuronal survival (13-18).

Previous studies have focused on the role and the alteration in the expression of MIP-3 $\alpha$ and CCR6 in response to pathological neuroinflammatory conditions, such as autoimmune 
encephalomyelitis, Alzheimer's disease, epilepsy and cerebral ischemia $(15,18-20)$. However, age-related alterations in the basal expression of MIP-3 $\alpha$ and CCR6 in the brain under normal condition have not yet been fully elucidated. Mongolian gerbils are considered to be a suitable model for studies on aging, as it has been indicated that age-dependent alterations in behavioral and biological processes in gerbils are similar to those in humans $(21,22)$. Therefore, the aim of the current study was to investigate age-dependent alterations in the protein expression of MIP-3 $\alpha$ and CCR6 in the hippocampus during the normal aging process in gerbils.

\section{Materials and methods}

Animals. In the present study, male Mongolian gerbils (Meriones unguiculatus) $(\mathrm{n}=36)$ at the age of postnatal month (PM) 1 (25-30 g) as the young, PM 6 (65-75 g) as the adult, PM 12 (75-85 g) as the middle-aged and PM 24 (85-95 g) as the aged group, were used. The gerbils ( $\mathrm{n}=9$ animals/group) were obtained from the Experimental Animal Center of Kangwon National University in the Republic of Korea. The gerbils were housed under conventional housing conditions at an ambient temperature of $23 \pm 3^{\circ} \mathrm{C}$, a relative humidity of $55 \pm 5 \%$ and a 12-h light/dark cycle. Free access to food and water was permitted. All experimental procedures including animal handling and use were reviewed and approved by the Institutional Animal Care and Use Committee of Kangwon National University (approval no. KW-200113-2).

Immunohistochemistry. For tissue preparation, all gerbils used in the present study were profoundly anaesthetized using an intraperitoneal injection of $60 \mathrm{mg} / \mathrm{kg}$ sodium pentobarbital, and cardiac perfusion was conducted. Confirmation of death was evaluated with vital signs including heart beats, pupillary response, and respiratory pattern (lack of cardiac activity for $5 \mathrm{~min}$ through cardiac palpation, unresponsiveness to light with dilated pupils using light shone into the eyes of the animal and lack of spontaneously breathing pattern with shallow and irregular breathing pattern). The gerbil brains were rinsed via transcardial perfusion of $0.1 \mathrm{M}$ PBS (pH 7.4) and fixed via perfusion of $4 \%$ paraformaldehyde in $0.1 \mathrm{M}$ PBS $20 \mathrm{~min}$ at room temperature. The brains were removed and post-fixed with the same fixative for $6 \mathrm{~h}$ at room temperature. The brain tissues including hippocampi were frontally sectioned to $30 \mu \mathrm{m}$ thickness using a cryostat at $-30^{\circ} \mathrm{C}$.

To examine age-related alterations in neurons, microglia, MIP-3 $\alpha$ and CCR6 in the hippocampus, immunohistochemical staining was performed according to a previous study (18). In brief, the sections were treated with $0.3 \%$ hydrogen peroxide $\left(\mathrm{H}_{2} \mathrm{O}_{2}\right)$ in PBS for $30 \mathrm{~min}$ and $10 \%$ normal goat serum (cat. no. S-1000, Vector Laboratories INC.) in $0.05 \mathrm{M}$ PBS for $30 \mathrm{~min}$. and incubated with mouse anti-neuronal nuclei (cat. no. MAB377, NeuN; 1:800; EMD Millipore) as a marker of neurons, rabbit anti-ionized calcium-binding adapter molecule 1 (cat. no. 019-19741, Iba1; 1:200; FUJIFILM Wako Pure Chemical Corporation) as a marker for microglia, rabbit anti-MIP-3 $\alpha$ (cat. no. ab9829, 1:50; Abcam) or rabbit anti-CCR6 (ab78429, 1:200; Abcam) for $10 \mathrm{~h}$ at $4^{\circ} \mathrm{C}$. Subsequently, the tissues were incubated with biotinylated horse anti-mouse (cat. no. BA-2001) or goat anti-rabbit (cat. no. BA-1000) IgG (1:200; Vector Laboratories Inc.) for $2 \mathrm{~h}$ at room temperature, and then to streptavidin peroxidase complex (cat. no. PK-7200, 1:200; Vector Laboratories Inc.) for $45 \mathrm{~min}$ at room temperature. Finally, the signal was visualized with 3,3'-diaminobenzidine.

For quantitative analysis of NeuN, Iba1, MIP-3 $\alpha$ and CCR6 immunoreactivity, six sections per animal with a $120-\mu \mathrm{m}$ thickness interval were selected. Digital images of NeuN, Iba1, MIP-3 $\alpha$ and CCR6 immunoreactive structures were captured at 20x magnification using an a light microscope (BX53, Olympus Corporation) equipped with a digital camera (Axiocam; Carl Zeiss AG), which was connected to a monitor. Firstly, the numbers of NeuN-immunoreactive neurons were counted in the target regions in the hippocampus, according to the method that was reported in a previous study (23). Briefly, NeuN-immunoreactive neurons were captured in a $250 \times 250 \mu \mathrm{m}$ region at the center of the stratum pyramidale of the hippocampus proper [cornu ammonis (CA)1-3 fields] and the granule cell layer of the dentate gyrus. The number of NeuN-immunoreactive neurons in each sample was quantified using Optimas v6.5 software (CyberMetrics Corporation). Secondly, according to a previously published method (18), the alterations in Iba-1 immunoreactivity were evaluated in the CA1-3 fields and the dentate gyrus. In addition, alterations in MIP-3 $\alpha$ and CCR6 immunoreactivity were evaluated in hippocampal cells as relative immunoreactivity (RI) using Image J v1.59 software (National Institutes of Health). Briefly, the images of Iba1, MIP-3 $\alpha$ and CCR6 were captured in the target cells or layers of the immunoreactive structures, calibrated to an array of $512 \times 512$ pixels and measured using a 0-255 grayscale system (white to dark signal corresponded to 255 to 0 ). After the background was subtracted, the staining intensity was calculated and expressed as RI. The ratio of RI in each immunoreactive structure was expressed as a percentage, with the PM 1 group designated as $100 \%$.

Statistical analysis. The data are presented as the mean \pm standard error of the mean. The differences in the mean RI among the groups were analyzed three times using one-way ANOVA followed by Bonferroni's multiple comparisons test using SPSS v17.0 software (SPSS, Inc.). $\mathrm{P}<0.05$ was considered to indicate a statistically significant difference.

\section{Results}

Alterations in NeuN immunoreactive neurons. In the PM 1, 6, 12 and 24 groups, NeuN immunoreactivity was primarily localized in neurons of the stratum pyramidale, which are called pyramidal neurons, in the hippocampal CA1-3 fields (Fig. 1A-D). The distribution pattern of NeuN immunoreactive neurons in the CA1-3 fields was similar in all groups. As in the hippocampus proper, the distribution pattern of NeuN immunoreactive neurons in the dentate gyrus did not differ between the groups (Fig. 1E-H). Furthermore, the numbers of NeuN immunoreactive neurons in both the CA1-3 fields and the dentate gyrus were similar in all groups (Fig. 1I). 

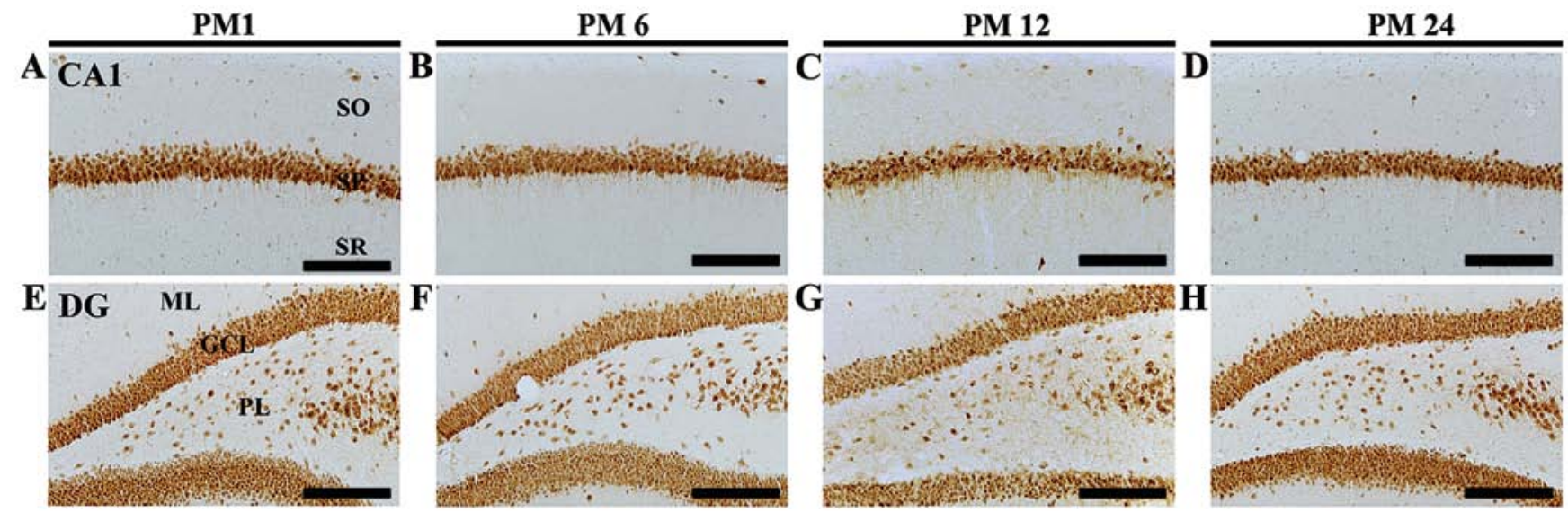

I

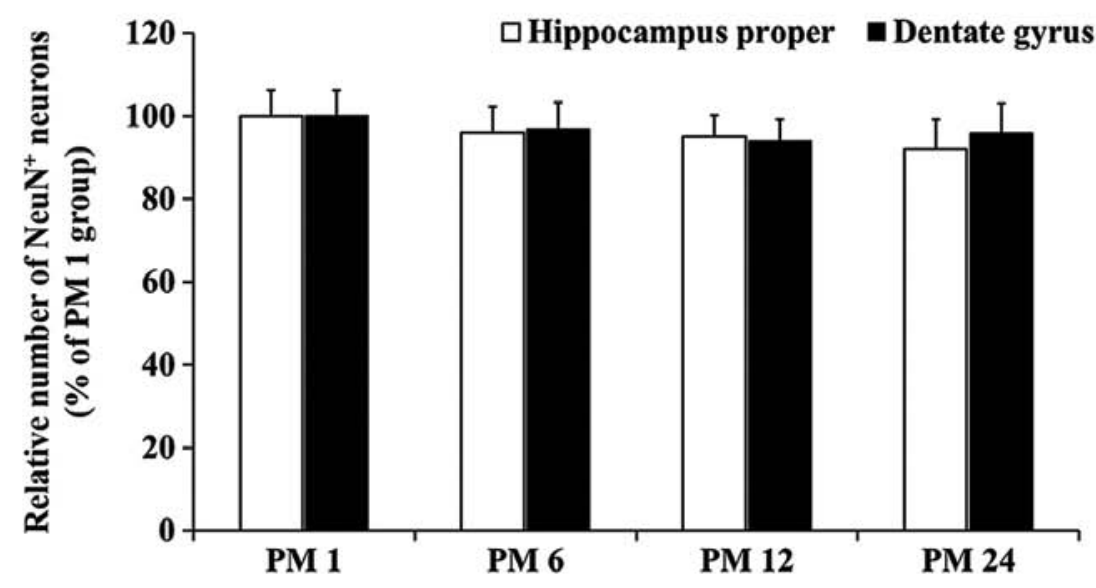

Figure 1. NeuN immunohistochemistry. NeuN immunoreactivity in the CA1 field in the (A) PM 1, (B) PM 6, (C) PM 12 and (D) PM 24 group, and in the DG in the (E) PM 1, (F) PM 6, (G) PM 12 and (H) PM 24 group. Scale bar=100 $\mu \mathrm{m}$. (I) Relative number of NeuN immunoreactive neurons in the SP and GCL of the PM 1, 6, 12 and 24 groups (n=9 per group). The data are presented as the mean \pm standard error of the mean. GCL, granule cell layer; ML, molecular layer; PL, polymorphic layer; SO, stratum oriens; SP, stratum pyramidale; SR, stratum radiatum; DG, dentate gyrus; CA, cornu ammonis; PM, postnatal month; NeuN anti-neuronal nuclei.

Alterations in Ibal immunoreactivity. In the PM 1 group, Iba1 immunoreactive microglia were detected in the stratum oriens and radiatum of the hippocampus proper (Fig. 2A). Iba1 immunoreactivity in the PM 6 group was decreased by $27.5 \%$ compared with the PM 1 group (Fig. $2 \mathrm{~B}$ and I). In the PM 12 group, Iba1 immunoreactivity was similar to that in the PM 1 group (Fig. 2C and I). However, in the PM 24 group, an increase of $25.9 \%$ in Iba1 immunoreactivity was observed compared with the PM 12 group (Fig. 2D and I). In this this group, certain Iba1 immunoreactive microglia were identified as activated microglia (enlarged cell bodies and thickened processes).

In the PM 1 group, Ibal immunoreactive microglia were primarily localized in the molecular and polymorphic layers of the dentate gyrus (Fig. 2E). Iba1 immunoreactivity in the PM 6 and 12 groups was decreased to 55.3 and $80.7 \%$, respectively, compared with the PM 1 group (Fig. 2F, G and I). In the PM 24 group, an increase of $35.9 \%$ in Iba1 immunoreactivity was observed compared with the PM 12 group (Fig. 2H and I).

Alterations in MIP-3a immunoreactivity. In the PM1 group, MIP-3 $\alpha$ immunoreactivity in the hippocampus proper was detected in pyramidal cells of the stratum pyramidale of the CA1-3 fields (Fig. 3A and E). In the PM 6 and 12 groups,
MIP-3 $\alpha$ immunoreactivity in pyramidal cells was decreased to 62.2 and $72.1 \%$, respectively, compared with the PM 1 group (Fig. 3B, C, F, G and M). A considerable increase in MIP-3 $\alpha$ immunoreactivity was observed in pyramidal cells of the PM 24 group, as RI was increased to $215.9 \%$ compared with the PM 1 group (Fig. 3D, $\mathrm{H}$ and $\mathrm{M}$ ).

In the PM 1 group, MIP-3 $\alpha$ in the dentate gyrus was detected primarily in granule cells of the granule cell layer (Fig. 3A and I). Similar to the hippocampus proper, MIP-3 $\alpha$ immunoreactivity in the dentate gyrus was decreased in the PM 6 and 12 groups to 85.9 and $88.9 \%$, respectively, compared with the PM 1 group (Fig. 3B, C and J-M). In the PM 24 group, MIP-3 $\alpha$ immunoreactivity was increased to $163.3 \%$ compared with the PM 1 group (Fig. 3D, L and M).

Alterations in CCR6 immunoreactivity. In the PM 1 group, a strong CCR6 immunoreactivity was primarily detected in the stratum pyramidale of the hippocampus proper (Fig. 4A and E). In the PM 6 and 12 groups, CCR6 immunoreactivity in the stratum pyramidale was faint (Fig. 4B, C, F, G and M). In the PM 24 group, CCR6 immunoreactivity in the stratum pyramidale was increased compared with the PM 6 and 12 groups, exhibiting an RI of $37.0 \%$ of that in the PM 1 group (Fig. 4D, H and M). 

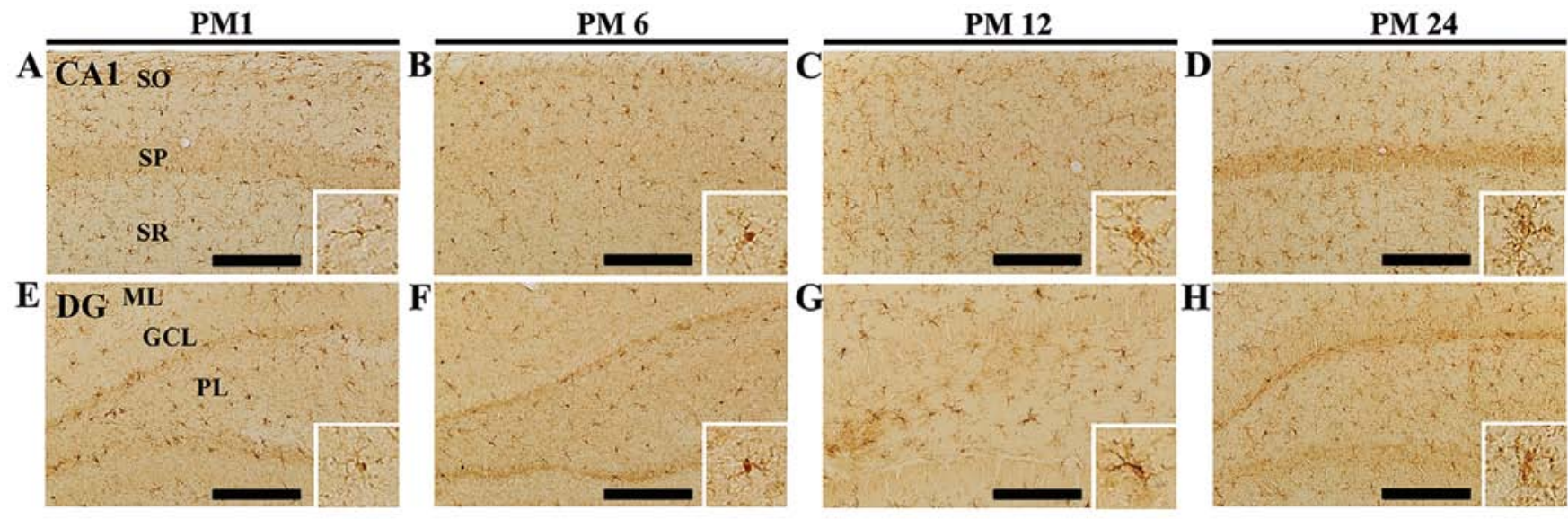

\section{I}

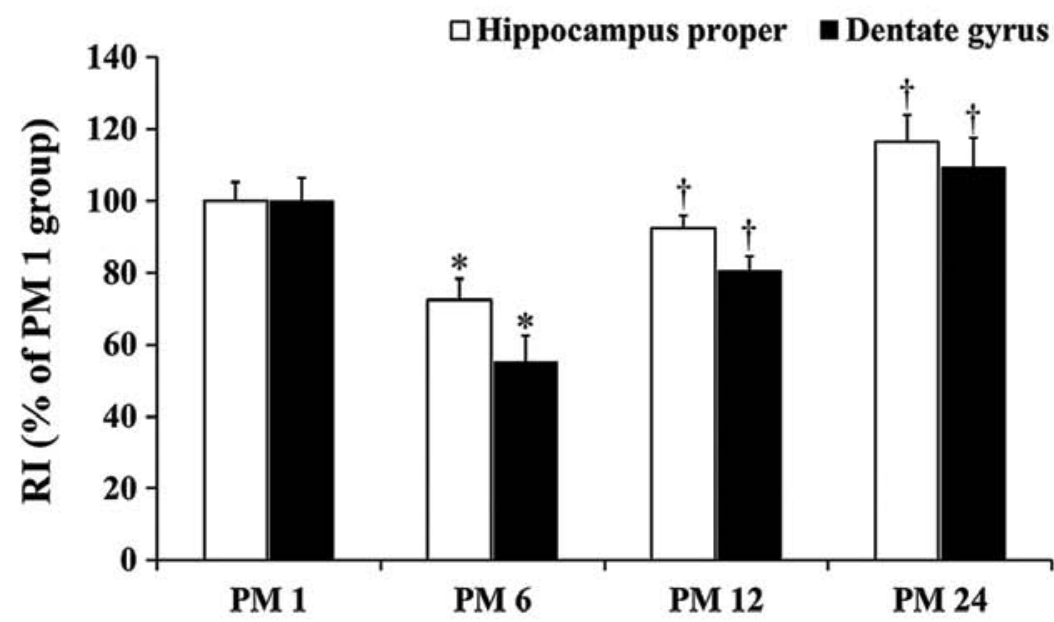

Figure 2. Iba-1 immunohistochemistry. Iba1 immunoreactivity in the CA1 field in the (A) PM 1, (B) PM 6, (C) PM 12 and (D) PM 24 group, and in the DG in the (E) PM 1, (F) PM 6, (G) PM 12 and (H) PM 24 group. Microglia in high magnification (x100) are presented in the smaller panels. Scale bar, $100 \mu \mathrm{m}$ (A-H). (I) RI as \% of Iba1 immunoreactivity in the CA1 field and the DG of the PM 1, 6, 12 and 24 groups (n=9 per group). The data are presented as the mean \pm standard error of the mean. "P<0.05 vs. PM 1; ${ }^{\mathrm{P}}<0.05$ vs. PM 6 and PM 12. GCL, granule cell layer; ML, molecular layer; PL, polymorphic layer; SO, stratum oriens; SP, stratum pyramidale; SR, stratum radiatum; RI, relative immunoreactivity; DG, dentate gyrus; CA, cornu ammonis; PM, postnatal month; Iba1, ionized calcium-binding adapter molecule 1 .

In the PM 1 group, a strong CCR6 immunoreactivity was also detected in the granule cell layer of the dentate gyrus (Fig. 4A and I). As in the case of the hippocampus proper, CCR6 immunoreactivity in the PM 6 and 12 groups was faint (Fig. 4B, C, J, K and M). In the PM 24 group, CCR6 immunoreactivity in the granule cell layer was increased compared with the PM 6 and 12 groups, with an RI of $19.9 \%$ of that in the PM 1 group (Fig. 4D, L and M).

\section{Discussion}

In the current study, the distribution pattern of NeuN immunoreactive neuronal cells in the hippocampus proper and dentate gyrus did not differ among the PM 1, 6, 12 and 24 groups, and the numbers of the neurons were indicated to be similar in all groups. This is in accordance with previous studies, which have reported that the neuronal number in the hippocampus proper and dentate gyrus in aged rodents was preserved (23-27). This finding indicates that the number of neurons in the rodent hippocampus does not alter during aging.

It has been reported that Iba1 immunoreactivity, which is a marker of microglia, was higher in the gerbil hippocampus in the young (PM 1) compared with the adult (PM 6) group (28). In the current study, Iba1 immunoreactivity in the hippocampus was decreased in the PM 6 and increased in the PM 24 group, compared with the PM 1 group. This finding indicates that neuroinflammation may be increased in the hippocampus during aging. Previous studies have revealed that microglia serve critical roles in neuroinflammation in the brain during normal aging, as they have been indicated to increase in numbers and size to acquire an active phenotype in the aged brains (28-31). Concomitantly with microglial activation, the expression of pro-inflammatory cytokines, such as interleukin (IL)-1 $\beta$, IL- 6 and tumor necrosis factor- $\alpha$ (TNF- $\alpha$ ), has also been indicated to alter in the hippocampus during the aging process. Balschun et al (32), reported that the basal mRNA expression of IL- $1 \beta$ was decreased in the hippocampus of middle-aged (12- to 16-month-old) compared with young (3-month-old) rats. On the contrary, Gavilan et al (3) reported that no significant differences were observed in the mRNA expression of IL- $1 \beta$ and TNF- $\alpha$ in the hippocampus between young and middle-aged rats. In addition, it has been indicated that the protein expression of cyclooxygenase- 2 in the hippocampus of the adult gerbil was decreased compared with the young group (33). By contrast, 

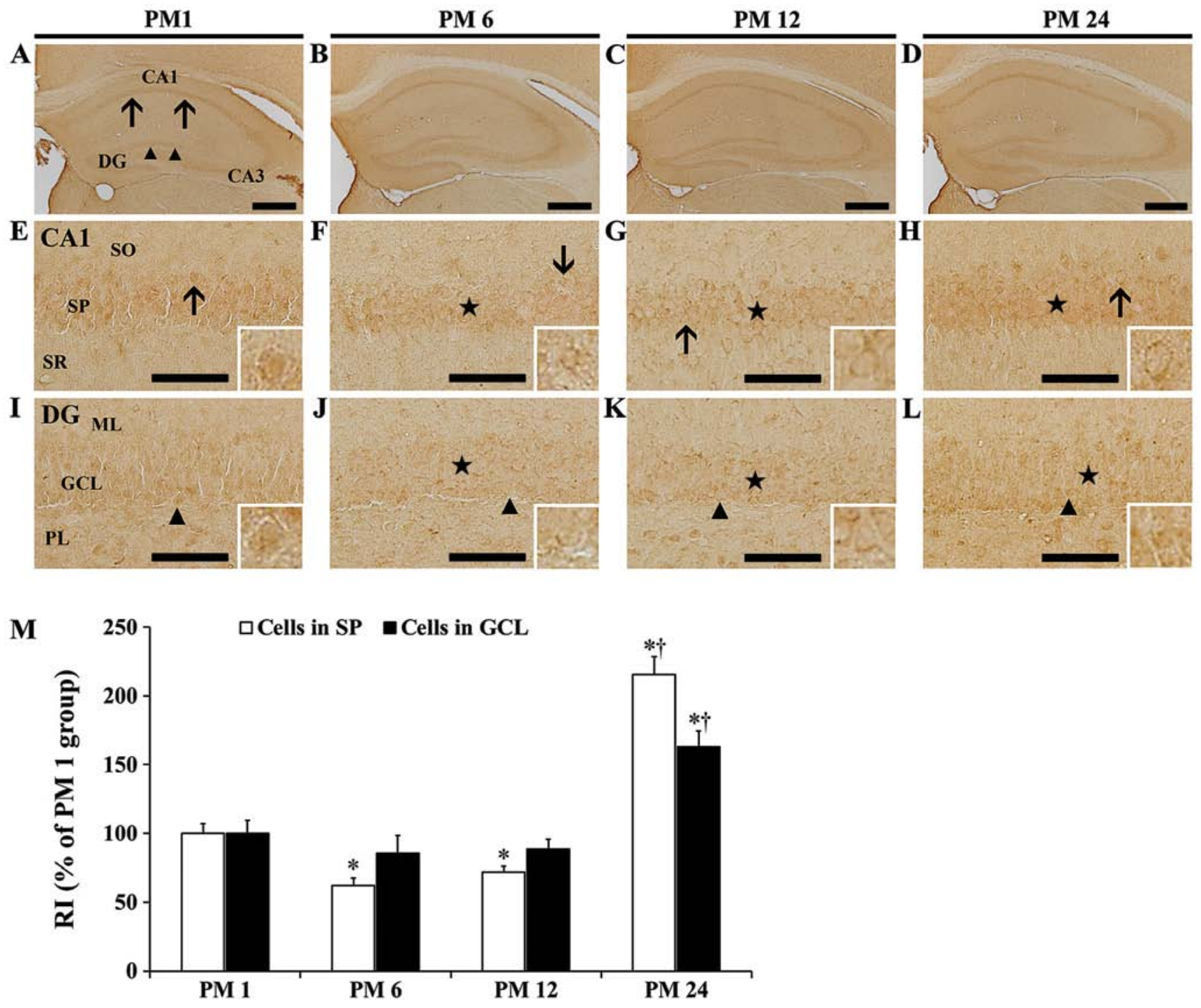

Figure 3. MIP-3 $\alpha$ immunohistochemistry. MIP-3 $\alpha$ immunoreactivity in the hippocampus in the (A) PM 1, (B) PM 6, (C) PM 12 and (D) PM 24 group, in the CA1 field in the (E) PM 1, (F) PM 6, (G) PM 12 and (H) PM 24 group, and in the DG in the (I) PM 1, (J) PM 6, (K) PM 12 and (L) PM 24 group. Scale bar, $400 \mu \mathrm{m}$ (A-C) and $50 \mu \mathrm{m}$ (D-I). (M) RI as \% of MIP-3 $\alpha$ immunoreactivity in the SP and GCL of the PM 1, 6, 12 and 24 groups (n=9 per group). The data are presented as the mean \pm standard error of the mean. ${ }^{*} \mathrm{P}<0.05$ vs. PM 1 ; ${ }^{\dagger} \mathrm{P}<0.05$ vs. PM 12. GCL, granule cell layer; ML, molecular layer; PL, polymorphic layer; SO, stratum oriens; SP, stratum pyramidale; SR, stratum radiatum; RI, relative immunoreactivity; DG, dentate gyrus; CA, cornu ammonis; PM, postnatal month; MIP-3 $\alpha$, macrophage inflammatory protein-3 $\alpha$.

the expression of pro-inflammatory cytokines, such as IL-1 $\beta$, IL- 6 , and TNF- $\alpha$, have been revealed to be increased in aged compared with young and middle-aged hippocampi in the normal aging process in rodents $(3,31,34)$. In addition, it has been reported that altered levels of cytokines and chemokines in aged brains were associated with an age-related cognitive decline (34-37). The age-related cognitive decline or impairment may be associated with the neuroinflammation that occurs in aged brains, and has been indicated to result in reductions in synaptic activity, pre-synaptic molecules, post-synaptic densities and dendritic and axonal arborization (3,38-40).

It has been reported that both MIP- $1 \alpha$ and MIP-1 $\beta$ were increased in the hippocampus of 30-month-old mice compared with the 4-month-old mice (41), suggesting that an age-dependent increase in chemokines may contribute to the decline of brain function during normal aging (41). In addition, it has been indicated that $\mathrm{C}-\mathrm{C}$ motif chemokine 2/monocyte chemoattractant protein-1 expression was increased in the aged mouse hippocampus (42). Furthermore, Subramanian et al (20) reported that CCR6 mRNA expression was increased in the brain of older symptomatic and pre-symptomatic 3xTg Alzheimer's disease mice and suggested that variations in the CCR6 level may be associated with alterations in cognition and learning behavior. In the current study, it was revealed that MIP-3 $\alpha$ and CCR6 immunoreactivities in the hippocampal cells of the PM 6 and 12 groups were decreased compared with the PM 1 group, while they were subsequently increased in the PM 24 group. This result is consistent with the findings by Liu et al (15), who suggested that CCR6 in pyramidal and granule cells may participate in regulating the activities of hippocampal principal neurons. In addition, it has been reported that MIP-3 $\alpha$ was increased in aged hippocampal principal cells concomitantly with inflammation, and CCR6 was increased in the neurons as a protective factor that is associated with 

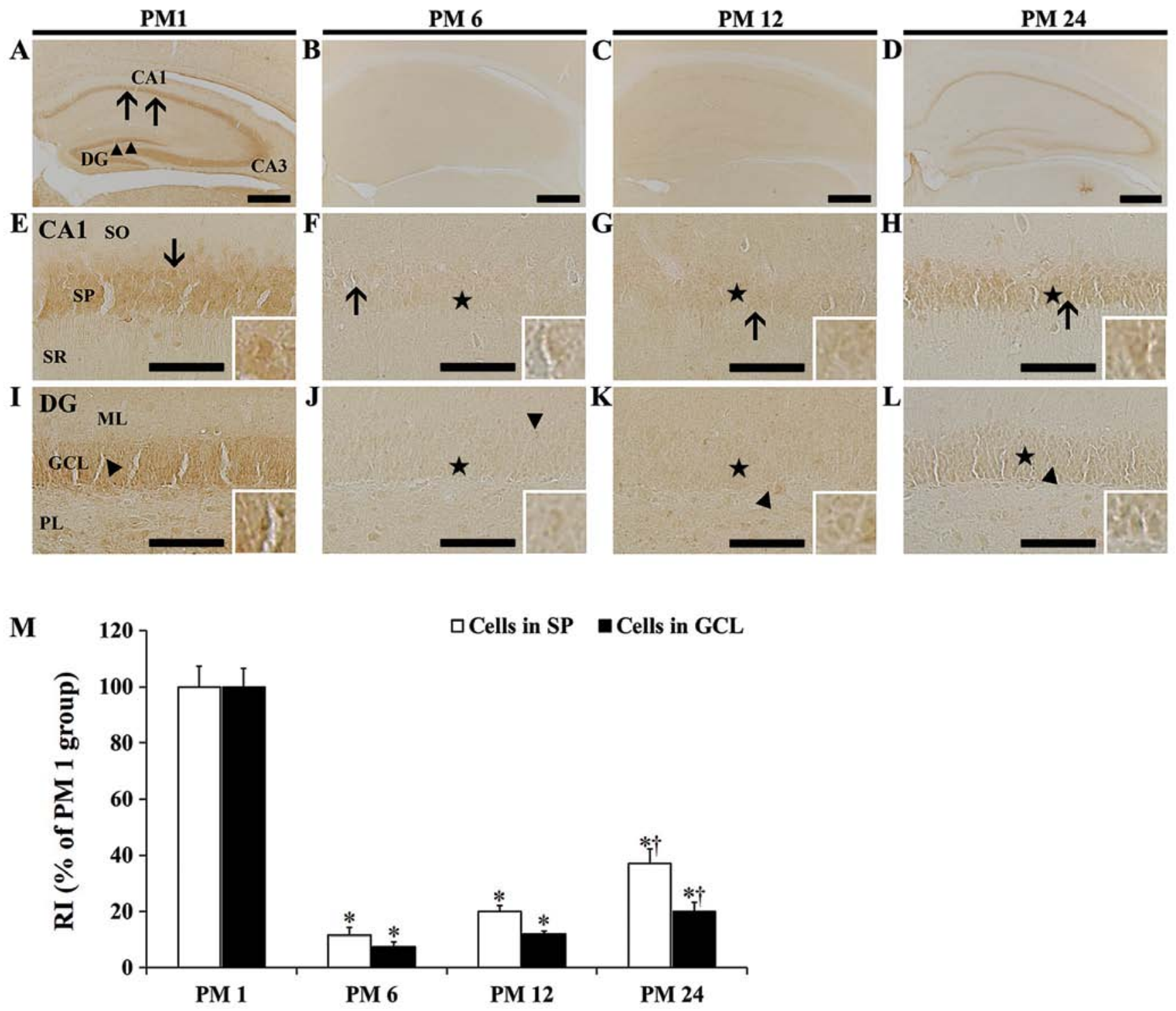

Figure 4. CCR6 immunohistochemistry. CCR6 immunoreactivity in the hippocampus in the (A) PM 1, (B) PM 6, (C) PM 12 and (D) PM 24 group, in the CA1 field in the (E) PM 1, (F) PM 6, (G) PM 12 and (H) PM 24 group, and in the DG in the (I) PM 1, (J) PM 6, (K) PM 12 and (L) PM 24 group. Scale bars, $400 \mu \mathrm{m}$ (A-C) and $50 \mu \mathrm{m}$ (D-I). (M) RI as \% of CCR6 immunoreactivity in the SP and GCL of the PM 1, 6, 12 and 24 groups (n=9 per group). The data are presented as the mean \pm standard error of the mean. ${ }^{*} \mathrm{P}<0.05$ vs. PM 1; ${ }^{\mathrm{P}}<0.05$ vs. PM 12. GCL, granule cell layer; ML, molecular layer; PL, polymorphic layer; SO, stratum oriens; SP, stratum pyramidale; SR, stratum radiatum; RI, relative immunoreactivity; DG, dentate gyrus; CA, cornu ammonis; PM, postnatal month; CCR6, C-C chemokine receptor type 6.

neuronal survival an animal model of status epilepticus and ischemic stroke $(15,18)$. Therefore, it may be hypothesized that an increased protein expression of MIP-3 $\alpha$ and CCR6 in the aged hippocampus may be associated with a decline in brain functions, including cognition and learning, during the normal aging process.

However, certain limitations exist to the present study. In the current study, age-dependent alterations in the protein expression of MIP-3 $\alpha$ and CCR6 in the hippocampal subregions were investigated during the normal aging process. Notably, the mechanisms via which MIP-3 $\alpha$ and CCR6 expression was regulated during the aging process, were not examined. Potential upstream regulators, which may affect MIP-3 $\alpha$ and CCR6 expression in the hippocampus at various aging stages, should be elucidated in future studies. In addition, the association between age-dependent alterations in neuroinflammation and the altered expression of MIP-3 $\alpha$ and CCR6 in aged animals should be addressed in future studies.

In conclusion, the current study revealed that MIP-3 $\alpha$ and CCR6 immunoreactivities in the gerbil hippocampus were altered during the normal aging process, and indicated that both MIP-3 $\alpha$ and CCR6 were decreased in the adult compared with the young hippocampus, and increased again in the aged compared with the adult hippocampus.

\section{Acknowledgements}

Not applicable.

\section{Funding}

The present study was funded by the Basic Science Research Program of the National Research Foundation (NRF) of 
Korea (grant nos. NRF-2017R1D1A1B03029311, NRF2017R1D1A1B03030161 and NRF- 2018R1D1A1B07049453).

\section{Availability of data and materials}

All data generated or analyzed during the current study are included in the published article.

\section{Authors' contributions}

CHL, JHA and JHP conceived the projects. JHA, JHP, MHW and CHL were responsible for the experimental design, data collection and manuscript writing. TKL, GEY, MCS and JHC performed the experiments, data analysis and critical comments on the present study. All authors have read and approved the final manuscript.

\section{Ethics approval and consent to participate}

All experimental procedures including animal handling and use were reviewed and approved by the Institutional Animal Care and Use Committee of Kangwon National University (approval no. KW-180124-2).

\section{Patient consent for publication}

Not applicable.

\section{Competing interests}

The authors declare that they have no competing interests.

\section{References}

1. Bettio LEB, Rajendran L and Gil-Mohapel J: The effects of aging in the hippocampus and cognitive decline. Neurosci Biobehav Rev 79: 66-86, 2017.

2. Geinisman Y, Detoledo-Morrell L, Morrell F and Heller RE: Hippocampal markers of age-related memory dysfunction: Behavioral, electrophysiological and morphological perspectives. Prog Neurobiol 45: 223-252, 1995.

3. Gavilan MP, Revilla E, Pintado C, Castaño A, Vizuete ML, Moreno-González I, Baglietto-Vargas D, Sánchez-Varo R, Vitorica J, Gutiérrez A and Ruano D: Molecular and cellular characterization of the age-related neuroinflammatory processes occurring in normal rat hippocampus: Potential relation with the loss of somatostatin GABAergic neurons. J Neurochem 103: 984-996, 2007.

4. Lee CH, Park JH, Choi JH, Yoo KY, Ryu PD and Won MH: Heat shock protein 90 and its cochaperone, p23, are markedly increased in the aged gerbil hippocampus. Exp Gerontol 46 : 768-772, 2011.

5. Lister JP and Barnes CA: Neurobiological changes in the hippocampus during normative aging. Arch Neurol 66: 829-833, 2009

6. Ojo B, Rezaie P, Gabbott PL, Davies H, Colyer F, CowleyTR, Lynch M and Stewart MG: Age-related changes in the hippocampus (loss of synaptophysin and glial-synaptic interaction) are modified by systemic treatment with an NCAM-derived peptide, FGL. Brain Behav Immun 26: 778-788, 2012.

7. Zlotnik A and Yoshie O: Chemokines: A new classification system and their role in immunity. Immunity 12: 121-127, 2000.

8. Bajetto A, Bonavia R, Barbero S and Schettini G: Characterization of chemokines and their receptors in the central nervous system: Physiopathological implications. J Neurochem 82: 1311-1329, 2002.

9. Cartier L, Hartley O, Dubois-Dauphin M and Krause KH: Chemokine receptors in the central nervous system: Role in brain inflammation and neurodegenerative diseases. Brain Res Brain Res Rev 48: 16-42, 2005.
10. Glabinski AR and Ransohoff RM: Chemokines and chemokine receptors in CNS pathology. J Neurovirol 5: 3-12, 1999.

11. Schilling M, Strecker JK, Ringelstein EB, Schabitz WR and Kiefer R: The role of CC chemokine receptor 2 on microglia activation and blood-borne cell recruitment after transient focal cerebral ischemia in mice. Brain Res 1289: 79-84, 2009.

12. Perez-Canadillas JM,Zaballos A, Gutierrez J, Varona R Roncal F Albar JP, Márquez G and Bruix M: NMR solution structure of murine CCL20/MIP-3alpha, a chemokine that specifically chemoattracts immature dendritic cells and lymphocytes through its highly specific interaction with the beta-chemokine receptor CCR6. J Biol Chem 276: 28372-28379, 2001.

13. Horuk R, Martin AW, Wang Z, Schweitzer L, Gerassimides A, Guo H, Lu Z, Hesselgesser J, Perez HD, Kim J, et al: Expression of chemokine receptors by subsets of neurons in the central nervous system. J Immunol 158: 2882-2890, 1997.

14. Lee JC, Ahn JH, Kim IH, Park JH, Yan BC, Cho GS, Ohk TH, Park CW, Cho JH, Kim YM, et al: Transient ischemia-induced change of CCR7 immunoreactivity in neurons and its new expression in astrocytes in the gerbil hippocampus. J Neurol Sci 336: 203-210, 2014.

15. Liu JX, Cao X, Liu Y and Tang FR: Altered expression of neuronal CCR6 during pilocarpine induced status epilepticus in mice. Epilepsy Res 126: 45-52, 2016.

16. Meucci O, Fatatis A, Simen AA, Bushell TJ, Gray PW and Miller RJ: Chemokines regulate hippocampal neuronal signaling and gp120 neurotoxicity. Proc Natl Acad Sci USA 95: 14500-14505, 1998.

17. Meucci O, Fatatis A, Simen AA and Miller RJ: Expression of CX3CR1 chemokine receptors on neurons and their role in neuronal survival. Proc Natl Acad Sci USA 97: 8075-8080, 2000.

18. Park JH, Noh Y, Kim SS, Ahn JH, Ohk TG, Cho JH, Lee TK, Kim H, Song M, Lee JC, et al: Time-course changes and new expressions of MIP-3 $\alpha$ and its receptor, CCR6, in the gerbil hippocampal CA1 area following transient global cerebral ischemia. Neurochem Res 43: 2102-2110, 2018.

19. Serafini B, Columba-Cabezas S, Di Rosa F and Aloisi F: Intracerebral recruitment and maturation of dendritic cells in the onset and progression of experimental autoimmune encephalomyelitis. Am J Pathol 157: 1991-2002, 2000.

20. Subramanian S, Ayala P, Wadsworth TL, Harris CL, Vandenbark AA, Quinn JF and Offner H: CCR6: A biomarker for Alzheimer's-like disease in a triple transgenic mouse model. J Alzheimers Dis 22: 619-629, 2010.

21. Cheal ML: The gerbil: A unique model for research on aging. Exp Aging Res 12: 3-21, 1986.

22. Vincent AL, Rodrick GE and Sodeman WA: The Mongolian gerbil in aging research. Exp Aging Res 6: 249-260, 1980.

23. Ahn JH, Choi JH, Park JH, Kim IH, Cho JH, Lee JC, Koo HM, Hwangbo G, Yoo KY Lee CH, et al: Long-term exercise improves memory deficits via restoration of myelin and microvessel damage, and enhancement of neurogenesis in the aged gerbil hippocampus after ischemic stroke. Neurorehabil Neural Repair 30: 894-905, 2016.

24. Ahn JH, Lee TK, Park JH, Cho JH, Kim IH, Lee JC, Hong S, Jeon YH, Kang Il J, Lee YJ, et al: Age-dependent differences in myelin basic protein expression in the hippocampus of young, adult and aged gerbils. Lab Anim Res 33: 237-243, 2017.

25. Rapp PR and Gallagher M: Preserved neuron number in the hippocampus of aged rats with spatial learning deficits. Proc Natl Acad Sci USA 93: 9926-9930, 1996.

26. West MJ: Regionally specific loss of neurons in the aging human hippocampus. Neurobiol Aging 14: 287-293, 1993.

27. Rasmussen T, Schliemann T, Sørensen JC,Zimmer J and West MJ: Memory impaired aged rats: No loss of principal hippocampal and subicular neurons. Neurobiol Aging 17: 143-147, 1996.

28. Choi JH, Lee CH, Hwang IK, Won MH, Seong JK, Yoon YS, Lee HS and Lee IS: Age-related changes in ionized calciumbinding adapter molecule 1 immunoreactivity and protein level in the gerbil hippocampal CA1 region. J Vet Med Sci 69: 1131-1136, 2007.

29. Cox FF, Carney D, Miller AM and Lynch MA: CD200 fusion protein decreases microglial activation in the hippocampus of aged rats. Brain Behav Immun 26: 789-796, 2012.

30. Lynch MA: Age-related neuroinflammatory changes negatively impact on neuronal function. Front Aging Neurosci 1: 6, 2010.

31. Ma Y, Matsuwaki T, Yamanouchi $\mathrm{K}$ and Nishihara M: Involvement of progranulin in modulating neuroinflammatory responses but not neurogenesis in the hippocampus of aged mice. Exp Gerontol 95: 1-8, 2017. 
32. Balschun D, Randolf A, Pitossi F, Schneider H, Del Rey A and Besedovsky HO: Hippocampal interleukin-1 beta gene expression during long-term potentiation decays with age. Ann N Y Acad Sci 992: 1-8, 2003.

33. Lee CH, Yoo KY, Choi JH, Park OK, Hwang IK, Kang Il J and Won MH: Cyclooxygenase-2 immunoreactivity and protein level in the gerbil hippocampus during normal aging. Neurochem Res 35: 99-106, 2010.

34. Griffin R, Nally R, Nolan Y, McCartney Y, Linden J and Lynch MA The age-related attenuation in long-term potentiation is associated with microglial activation. J Neurochem 99: 1263-1272, 2006.

35. Scheinert RB, Asokan A, Rani A, Kumar A, Foster TC and Ormerod BK: Some hormone, cytokine and chemokine levels that change across lifespan vary by cognitive status in male Fischer 344 rats. Brain Behav Immun 49: 216-232, 2015.

36. Speisman RB, Kumar A, Rani A, Foster TC and Ormerod BK Daily exercise improves memory, stimulates hippocampal neurogenesis and modulates immune and neuroimmune cytokines in aging rats. Brain Behav Immun 28: 25-43, 2013.

37. Villeda SA, Plambeck KE, Middeldorp J, Castellano JM Mosher KI, Luo J, Smith LK, Bieri G, Lin K, Berdnik D, et al: Young blood reverses age-related impairments in cognitive function and synaptic plasticity in mice. Nat Med 20: 659-663, 2014.
38. Chung HY, Cesari M, Anton S, Marzetti E, Giovannini S, Seo AY, Carter C, Yu BP and Leeuwenburgh C: Molecular inflammation: Underpinnings of aging and age-related diseases. Ageing Res Rev 8: 18-30, 2009.

39. Tha KK, Okuma Y, Miyazaki H, Murayama T, Uehara T, Hatakeyama R, Hayashi Y and Nomura Y: Changes in expressions of proinflammatory cytokines IL-1beta, TNF-alpha and IL-6 in the brain of senescence accelerated mouse (SAM) P8. Brain Res 885: 25-31, 2000.

40. Yankner BA, Lu T and Loerch P: The aging brain. Annu Rev Pathol 3: 41-66, 2008

41. Felzien LK, McDonald JT, Gleason SM, Berman NE and Klein RM: Increased chemokine gene expression during aging in the murine brain. Brain Res 890: 137-146, 2001.

42. Terao A, Apte-Deshpande A, Dousman L, Morairty S, Eynon BP, Kilduff TS and Freund YR: Immune response gene expression increases in the aging murine hippocampus. J Neuroimmunol 132: 99-112, 2002.

This work is licensed under a Creative Commons Attribution-NonCommercial-NoDerivatives 4.0 International (CC BY-NC-ND 4.0) License. 